NONCOMMUTATIVE GEOMETRY

AND QUANTUM GROUPS

BANACH CENTER PUBLICATIONS, VOLUME 61

INSTITUTE OF MATHEMATICS

POLISH ACADEMY OF SCIENCES

WARSZAWA 2003

\title{
THE CORIBBON STRUCTURES OF SOME FINITE DIMENSIONAL BRAIDED HOPF ALGEBRAS GENERATED BY $2 \times 2$-MATRIX COALGEBRAS
}

\author{
MICHIHISA WAKUI \\ Department of Mathematics, Osaka University, Toyonaka, Osaka 560-0043, Japan \\ E-mail: wakui@math.sci.osaka-u.ac.jp
}

\begin{abstract}
We determine the coribbon structures of some finite dimensional braided Hopf algebras generated by $2 \times 2$-matrix coalgebras constructed by S. Suzuki. As a consequence, we see that such a Hopf algebra has a coribbon structure if and only if it is of Kac-Paljutkin type.
\end{abstract}

1. Introduction. Finite dimensional Hopf algebras with some additional structures such as $R$-matrices, braidings and ribbon elements are closely related to the topology of knots, links and 3-manifolds. For example, it is shown by Kauffman [6] that many basic knot invariants including the Alexander-Conway polynomial and the Jones polynomial are derived from spin-preserving $R$-matrices $R: \mathrm{M}_{2}(\mathbf{k}) \otimes \mathrm{M}_{2}(\mathbf{k}) \rightarrow \mathrm{M}_{2}(\mathbf{k}) \otimes \mathrm{M}_{2}(\mathbf{k})$, where $\mathrm{M}_{2}(\mathbf{k})$ is the $2 \times 2$ matrix algebra over a field $\mathbf{k}$. Another type of $R$-matrices $R: \mathrm{M}_{2}(\mathbf{k}) \otimes \mathrm{M}_{2}(\mathbf{k}) \rightarrow \mathrm{M}_{2}(\mathbf{k}) \otimes \mathrm{M}_{2}(\mathbf{k})$ are classified and investigated by Takeuchi and Tambara [14], Radford [9] and S. Suzuki [11] (see also [13]). In particular, S. Suzuki [11] introduced a family of cosemisimple Hopf algebras of finite dimension, and determined the (co)quasitriangular structures of those Hopf algebras, by classifying and investigating $R$-matrices of type $\left(\begin{array}{cccc}0 & 0 & 0 & \alpha \\ 0 & \beta & 0 & 0 \\ 0 & 0 & \beta & 0 \\ \alpha & 0 & 0 & 0\end{array}\right)$, where $\alpha, \beta \in \mathbf{k}$ with $\alpha, \beta \neq 0$. The family includes Hopf algebras $K$ which fit into a Hopf algebra extension $1 \rightarrow\left(\mathbf{k} C_{2}\right)^{*} \rightarrow K \rightarrow \mathbf{k} D_{2 L} \rightarrow 1$, where $C_{2}$ is the cyclic group of order 2 and $D_{2 L}$ is the dihedral group of order $2 L$ (see $[4,7,8,11]$ for more precise information). So, such a Hopf algebra $K$ can be said to be of Kac-Paljutkin type, as a generalization of the 8-dimensional Hopf algebra [5] defined by them.

Reshetikhin and Turaev [10] introduced the notion of a ribbon Hopf algebra to give applications to knot and 3-manifold theory. The notion of a coribbon Hopf algebra was

2000 Mathematics Subject Classification: Primary 16W30; Secondary 57M27.

Key words and phrases: Hopf algebras, quasitriangular structures, coribbon Hopf algebras, $R$-matrices, quadratic bialgebras, invariants of knots and 3-manifolds.

The paper is in final form and no version of it will be published elsewhere. 
introduced by Hayashi [3] as a dual notion to that of a ribbon Hopf algebra. He classifies the braidings and ribbon structures on quantized classical groups and on some Hopf face algebras which are closely related to the $S U(N)_{L}$-topological quantum field theories.

In this article, using the universal property of quadratic bialgebras [1] with respect to coribbon structures, we determine the coribbon structures of the braided cosemisimple Hopf algebras constructed by Suzuki. As a consequence, we see that such a Hopf algebra has a coribbon structure if and only if it is of Kac-Paljutkin type (see Theorem 5).

This article consists of three sections. In Section 2, we review definitions and results about braided bialgebras and quadratic bialgebras due to Doi [1], and show that any central element of a Yang-Baxter coalgebra $(C, \sigma)$ can be extended to a coribbon element of the quadratic bialgebra $M(C, \sigma)$. In Section 3, we determine the coribbon structures of the cosemisimple Hopf algebras constructed by Suzuki.

For a Hopf algebra $A, \Delta, \varepsilon$ and $S$ denote the comultiplication, the counit and the antipode of $A$, respectively. We use Sweedler's notation $\Delta(x)=\sum x^{(1)} \otimes x^{(2)}$ for $x \in A$ [12]. We work over a fixed field $\mathbf{k}$, and set $\mathbf{k}^{\times}:=\mathbf{k}-\{0\}$. For $f \in \operatorname{Hom}_{\mathbf{k}}(C, A)$, where $C$ is a coalgebra and $A$ is an algebra, we denote by $f^{-1}$ the inverse with respect to the convolution product, and call it the inverse of $f$, simply. For a finite dimensional vector space $V$, the dual vector $\operatorname{space}^{\operatorname{Hom}_{\mathbf{k}}}(V, \mathbf{k})$ is denoted by $V^{*}$.

2. Coribbon structures of quadratic bialgebras. Doi [1] showed that any YangBaxter form $\sigma$ on a coalgebra $C$ can be uniquely extended to a braiding $\tilde{\sigma}$ of the quadratic bialgebra $M(C, \sigma)$. Such a universal property holds for a central element of the YangBaxter coalgebra $(C, \sigma)$. More precisely, any central element of a Yang-Baxter coalgebra $(C, \sigma)$ can be uniquely extended to a coribbon element of the braided bialgebra $(M(C, \sigma), \tilde{\sigma})$. In this section, we show such a universal property.

Definition 1. Let $B$ be a bialgebra over $\mathbf{k}$ and $\sigma: B \otimes B \rightarrow \mathbf{k}$ an invertible bilinear form. The bilinear form $\sigma$ is said to be a braiding on $B$ if the following three conditions are satisfied:

(i) $\sum \sigma\left(x^{(1)}, y^{(1)}\right) x^{(2)} y^{(2)}=\sum y^{(1)} x^{(1)} \sigma\left(x^{(2)}, y^{(2)}\right)$ for all $x, y \in B$.

(ii) $\sigma(x y, z)=\sum \sigma\left(x, z^{(1)}\right) \sigma\left(y, z^{(2)}\right)$ for all $x, y, z \in B$.

(iii) $\sigma(x, y z)=\sum \sigma\left(x^{(1)}, z\right) \sigma\left(x^{(2)}, y\right)$ for all $x, y, z \in B$.

We call such a pair $(B, \sigma)$ a braided bialgebra.

Definition 2. Let $C$ be a coalgebra over $\mathbf{k}$ and $\sigma: C \otimes C \rightarrow \mathbf{k}$ an invertible bilinear form. The bilinear form $\sigma$ is said to be a Yang-Baxter form if for all $x, y, z \in C$, the following equation holds:

$$
\sum \sigma\left(x^{(1)}, y^{(1)}\right) \sigma\left(x^{(2)}, z^{(1)}\right) \sigma\left(y^{(2)}, z^{(2)}\right)=\sum \sigma\left(y^{(1)}, z^{(1)}\right) \sigma\left(x^{(1)}, z^{(2)}\right) \sigma\left(x^{(2)}, y^{(2)}\right) .
$$

We call such a pair $(C, \sigma)$ a Yang-Baxter coalgebra.

Let $C$ be a coalgebra over $\mathbf{k}$ and $\sigma \in(C \otimes C)^{*}$ be invertible. We define the coideal $I_{\sigma}$ of the tensor bialgebra $\mathcal{T}(C)[12]$ by

$$
I_{\sigma}:=\left\{\sum \sigma\left(x^{(1)}, y^{(1)}\right) x^{(2)} y^{(2)}-\sum y^{(1)} x^{(1)} \sigma\left(x^{(2)}, y^{(2)}\right) \mid x, y, z \in C\right\} .
$$


Then, we get a bialgebra $M(C, \sigma):=\mathcal{T}(C) /\left\langle I_{\sigma}\right\rangle$. This bialgebra is called the quadratic bialgebra associated with $(C, \sigma)$.

In the theory of braided bialgebras, the following result due to Doi [1] is fundamental.

Theorem 1 (Doi). Let $(C, \sigma)$ be a Yang-Baxter coalgebra over $\mathbf{k}$. Then there is a unique $\mathbf{k}$-linear form $\tilde{\sigma}: M(C, \sigma) \otimes M(C, \sigma) \rightarrow \mathbf{k}$ such that

(i) $(M(C, \sigma), \tilde{\sigma})$ is a braided bialgebra.

(ii) $\tilde{\sigma}(x, y)=\sigma(x, y)$ for all $x, y \in C$.

Here, we naturally identified $x \in C$ and its image under the canonical injection $C \rightarrow$ $M(C, \sigma)$.

The notion of a coribbon Hopf algebra is due to Hayashi [3].

Definition 3. Let $(A, \sigma)$ be a braided bialgebra over k. An invertible element $\theta \in A^{*}$ is said to be a coribbon element of $(A, \sigma)$ if the following three conditions are satisfied.

(i) $\sum \theta\left(x^{(1)}\right) x^{(2)}=\sum x^{(1)} \theta\left(x^{(2)}\right) \quad$ for all $x \in A$.

(ii) $\theta(1)=1$.

(iii) $\theta(x y)=\sum \sigma^{-1}\left(x^{(1)}, y^{(1)}\right) \theta\left(x^{(2)}\right) \theta\left(y^{(2)}\right) \sigma^{-1}\left(y^{(3)}, x^{(3)}\right) \quad$ for all $x, y \in A$.

We call such a triple $(A, \sigma, \theta)$ a balanced braided bialgebra. When $A$ is a Hopf algebra, we require the following condition in addition to the above conditions.

(iv) $\theta \circ S=\theta$.

We call the triple $(A, \sigma, \theta)$ satisfying the conditions (i), (ii), (iii) and (iv) a balanced braided Hopf algebra or a coribbon Hopf algebra.

REMARK. If the dimension of a Hopf algebra $A$ is finite, then under the identification $(A \otimes A)^{*} \cong A^{*} \otimes A^{*}$, a braiding $\sigma$ is a universal $R$-matrix of $A^{*}[2]$ and a coribbon element $\theta$ is a ribbon element of $\left(A^{*}, \sigma\right)$ in the sense of Reshetikhin and Turaev [10].

As an analogue of Theorem 1, we have the following theorem.

Theorem 2. Let $(C, \sigma)$ be a Yang-Baxter coalgebra over $\mathbf{k}$ and $\theta \in C^{*}$ an invertible element. If $\theta$ satisfies the equation

$$
\sum \theta\left(x^{(1)}\right) x^{(2)}=\sum x^{(1)} \theta\left(x^{(2)}\right) \text { for all } x \in C,
$$

then there is a unique $\mathbf{k}$-linear form $\tilde{\theta}: M(C, \sigma) \rightarrow \mathbf{k}$ such that

(i) $(M(C, \sigma), \tilde{\sigma}, \tilde{\theta})$ is a balanced braided bialgebra.

(ii) $\tilde{\theta}(x)=\theta(x)$ for all $x \in C$.

Here, $\tilde{\sigma}$ is the braiding of $M(C, \sigma)$ as in Theorem 1.

Proof. The Yang-Baxter operator $\sigma$ can be extended uniquely to $\sigma: \mathcal{T}(C) \otimes \mathcal{T}(C) \rightarrow \mathbf{k}$ such that

$$
\begin{array}{ll}
\sigma(x y, z)=\sum \sigma\left(x, z^{(1)}\right) \sigma\left(y, z^{(2)}\right), & \forall x, y, z \in \mathcal{T}(C) \\
\sigma(x, y z)=\sum \sigma\left(x^{(1)}, z\right) \sigma\left(x^{(2)}, y\right), \quad \forall x, y, z \in \mathcal{T}(C) .
\end{array}
$$

This linear form $\sigma: \mathcal{T}(C) \otimes \mathcal{T}(C) \rightarrow \mathbf{k}$ induces a braiding on $M(C, \sigma)$ [1]. Hence, for $x, y, z \in \mathcal{T}(C)$, we have 


$$
\sum \sigma\left(x^{(1)}, y^{(1)}\right) \sigma\left(x^{(2)}, z^{(1)}\right) \sigma\left(y^{(2)}, z^{(2)}\right)=\sum \sigma\left(y^{(1)}, z^{(1)}\right) \sigma\left(x^{(1)}, z^{(2)}\right) \sigma\left(x^{(2)}, y^{(2)}\right)
$$

Using this equation, we see that $\theta$ and its inverse $\theta^{-1}$ can be extended uniquely to $\theta: \mathcal{T}(C) \rightarrow \mathbf{k}$ and $\theta^{-1}: \mathcal{T}(C) \rightarrow \mathbf{k}$ such that

$$
\begin{aligned}
\theta(x y) & =\sum \sigma^{-1}\left(x^{(1)}, y^{(1)}\right) \theta\left(x^{(2)}\right) \theta\left(y^{(2)}\right) \sigma^{-1}\left(y^{(3)}, x^{(3)}\right), \quad \forall x, y \in \mathcal{T}(C), \\
\theta^{-1}(x y) & =\sum \sigma\left(y^{(1)}, x^{(1)}\right) \theta^{-1}\left(x^{(2)}\right) \theta^{-1}\left(y^{(2)}\right) \sigma\left(x^{(3)}, y^{(3)}\right), \quad \forall x, y \in \mathcal{T}(C) .
\end{aligned}
$$

These linear forms on $\mathcal{T}(C)$ are inverses of each other, and satisfy $\theta\left(I_{\sigma}\right)=\theta^{-1}\left(I_{\sigma}\right)=0$. Therefore, $\theta: \mathcal{T}(C) \rightarrow \mathbf{k}$ induces an invertible linear form $\tilde{\theta}: M(C, \sigma) \rightarrow \mathbf{k}$. Since

$$
\sum y^{(1)} x^{(1)} \tilde{\sigma}^{-1}\left(y^{(2)}, x^{(2)}\right)=\sum \tilde{\sigma}^{-1}\left(y^{(1)}, x^{(1)}\right) x^{(2)} y^{(2)} \quad \text { for } x, y \in M(C, \sigma),
$$

we see that the linear form $\tilde{\theta}$ satisfies the equation

$$
\sum \tilde{\theta}\left(x^{(1)}\right) x^{(2)}=\sum x^{(1)} \tilde{\theta}\left(x^{(2)}\right), \text { for all } x \in M(C, \sigma) .
$$

It is clear that $\tilde{\theta}$ satisfies the conditions (ii) and (iii). Thus, $(M(C, \sigma), \tilde{\sigma}, \tilde{\theta})$ is a balanced braided bialgebra.

Since the bialgebra $M(C, \sigma)$ is generated by $C$, any coribbon element on $M(C, \sigma)$ is uniquely determined on $C$. It follows that a coribbon element of $(M(C, \sigma), \tilde{\sigma})$ is unique. This completes the proof.

Corollary 3. Let $(A, \sigma)$ be a braided bialgebra over $\mathbf{k}$. Suppose that $A$ is generated by sub-coalgebra $C$ as an algebra, and $\theta \in C^{*}$ is an invertible element such that

$$
\sum \theta\left(x^{(1)}\right) x^{(2)}=\sum x^{(1)} \theta\left(x^{(2)}\right) \text { for all } x \in C
$$

Then the following are equivalent.

(i) There exists a coribbon element $\theta^{\prime} \in A^{*}$ such that $\left.\theta^{\prime}\right|_{C}=\theta$.

(ii) $\tilde{\theta}($ Kerĩ $)=0$, where $\tilde{\theta}$ is the coribbon element of the braided bialgebra $\left(M\left(C,\left.\sigma\right|_{C \otimes C}\right)\right.$, $\widetilde{\sigma \mid} \otimes C)$.

Here, $\tilde{\iota}: M\left(C,\left.\sigma\right|_{C \otimes C}\right) \rightarrow A$ is the bialgebra homomorphism induced from the inclusion $\iota: C \hookrightarrow A$. Under the above situation, we have

$$
\theta^{\prime} \circ \tilde{\iota}=\tilde{\theta} .
$$

Proof. (i) $\Rightarrow$ (ii): Let $\theta^{\prime} \in A^{*}$ be a coribbon element of $(A, \sigma)$ such that $\left.\theta^{\prime}\right|_{C}=\theta$. It is enough to show that $\theta^{\prime} \circ \tilde{\iota}=\tilde{\theta}$. This follows from the following commutative diagram.

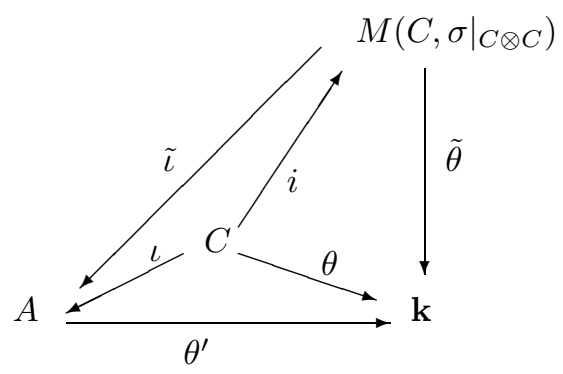

Here, $i: C \rightarrow M\left(C,\left.\sigma\right|_{C \otimes C}\right)$ is the canonical injection. 
(ii) $\Rightarrow$ (i): Since $\tilde{\theta}(\operatorname{Ker} \tilde{\iota})=0$, there exists a k-linear form $\bar{\theta}$ on $M\left(C,\left.\sigma\right|_{C \otimes C}\right) /$ Ker $\tilde{\iota}$ such that $\tilde{\theta}=\bar{\theta} \circ \pi$, where $\pi: M\left(C,\left.\sigma\right|_{C \otimes C}\right) \rightarrow M\left(C,\left.\sigma\right|_{C \otimes C}\right) / \operatorname{Ker} \tilde{\iota}$ is the natural projection. Since $\tilde{\iota}$ is a bialgebra homomorphism, Ker $\tilde{\iota}$ is a bi-ideal of $M\left(C,\left.\sigma\right|_{C \otimes C}\right)$. Thus, $\tilde{\theta}^{-1}($ Ker $\tilde{\iota})=0$, and whence $\bar{\theta}: M\left(C,\left.\sigma\right|_{C \otimes C}\right) /$ Ker $\tilde{\iota} \rightarrow \mathbf{k}$ is invertible with respect to the convolution product.

Let $\tau: M\left(C,\left.\sigma\right|_{C \otimes C}\right) / \operatorname{Ker} \tilde{\iota} \otimes M\left(C,\left.\sigma\right|_{C \otimes C}\right) /$ Ker $\tilde{\iota} \rightarrow \mathbf{k}$ be the $\mathbf{k}$-linear form such that

$$
\tau \circ(\pi \otimes \pi)=\widetilde{\sigma \Gamma_{C \otimes C}}
$$

Then $M(C, \sigma) / \operatorname{Ker} \tilde{\iota}$ is a braiding, and $\bar{\theta}$ is a coribbon element of $\left(M\left(C,\left.\sigma\right|_{C \otimes C}\right) / \operatorname{Ker} \tilde{\iota}, \tau\right)$. Since $A$ is generated by $C, \tilde{\iota}$ is surjective. Thus,

$$
M\left(C,\left.\sigma\right|_{C \otimes C}\right) / \operatorname{Ker} \tilde{\iota} \cong A \text { as bialgebras. }
$$

Regarding $\bar{\theta}: M\left(C,\left.\sigma\right|_{C \otimes C}\right) /$ Ker $\tilde{\iota} \rightarrow \mathbf{k}$ as a linear mapping from $A$ to $\mathbf{k}$ through the above isomorphism, we have a linear form $\theta^{\prime}: A \rightarrow \mathbf{k}$ such that $\theta^{\prime} \circ \tilde{\iota}=\tilde{\theta}$.

Since $\sigma \circ(\tilde{\iota} \otimes \tilde{\iota})=\tilde{\sigma}$, the bilinear form on $A \otimes A$ corresponding to $\tau$ coincides with $\sigma$. Hence, $\theta^{\prime}$ is a coribbon element of $(A, \sigma)$.

3. The coribbon structures of $A_{N L}^{\nu \lambda}$. Suzuki [11] introduced a family of cosemisimple Hopf algebras of finite dimension, and determined the (co)quasitriangular structures of those Hopf algebras. In this section, first of all, we review Suzuki's results, and then determine the coribbon structures of those Hopf algebras. Throughout this section, $\mathbf{k}$ denotes an algebraically closed field of $\operatorname{char}(\mathbf{k}) \neq 2$.

Let $C$ be the dual coalgebra of the $2 \times 2$-matrix algebra $\mathrm{M}_{2}(\mathbf{k})$. Then, $C$ has a basis $\left\{X_{11}, X_{12}, X_{21}, X_{22}\right\}$ such that

$$
\Delta\left(X_{i j}\right)=X_{i 1} \otimes X_{1 j}+X_{i 2} \otimes X_{2 j}, \quad \varepsilon\left(X_{i j}\right)=\delta_{i j} \quad(i, j=1,2) .
$$

We define the coideal $I$ of the tensor bialgebra $\mathcal{T}(C)$ by

$$
I=\mathbf{k}\left(X_{11}^{2}-X_{22}^{2}\right)+\mathbf{k}\left(X_{12}^{2}-X_{21}^{2}\right)+\sum_{i-j \not \equiv-m(\bmod 2)} \mathbf{k}\left(X_{i j} X_{l m}\right),
$$

and denote by $B$ the quotient bialgebra obtained from $\mathcal{T}(C)$ divided by the bi-ideal $\langle I\rangle$ generated by $I$. We write $x_{i j}$ for the image of $X_{i j}$ under the natural projection $\mathcal{T}(C) \rightarrow B$.

Let $N \geq 1, L \geq 2$ and $\nu, \lambda= \pm 1$. Then, we have a coideal $J_{N L}^{\nu \lambda}$ of $B$ defined by

$$
\begin{aligned}
J_{N L}^{\nu \lambda}: & =\mathbf{k}\left(x_{11}^{2 N}+\nu x_{12}^{2 N}-1\right)+\mathbf{k}(\underbrace{x_{11} x_{22} x_{11} \cdots \cdots}_{L}-\underbrace{x_{22} x_{11} x_{22} \cdots \cdots}_{L}) \\
& +\mathbf{k}(-\lambda \underbrace{x_{12} x_{21} x_{12} \cdots \cdots}_{L}+\underbrace{x_{21} x_{12} x_{21} \cdots \cdots}_{L})
\end{aligned}
$$

Here,

$$
\underbrace{x_{11} x_{22} x_{11} \cdots \cdots}_{L}= \begin{cases}\left(x_{11} x_{22}\right)^{\frac{L}{2}} & \text { if } L \text { is even } \\ \left(x_{11} x_{22}\right)^{\frac{L-1}{2}} x_{11} & \text { if } L \text { is odd }\end{cases}
$$

and so on. We set

$$
A_{N L}^{\nu \lambda}:=B /\left\langle J_{N L}^{\nu \lambda}\right\rangle
$$


The quotient bialgebra $A_{N L}^{\nu \lambda}$ has a unique Hopf algebra structure such that $C$ is a subcoalgebra. We write the image of $x_{i j}$ under the natural projection $\pi: B \rightarrow A_{N L}^{\nu \lambda}$ by the same notation. Then the set

$$
\{x_{11}^{s} \overbrace{x_{22} x_{11} x_{22} \cdots \cdots}^{t}, x_{12}^{s} \overbrace{x_{21} x_{12} x_{21} \cdots \cdots}^{t} \mid 1 \leq s \leq 2 N, 0 \leq t \leq L-1\}
$$

is a basis over $\mathbf{k}$ of $A_{N L}^{\nu \lambda}$ [11]. Therefore, we see that $\operatorname{dim} A_{N L}^{\nu \lambda}=4 N L$.

REMARK. The Hopf algebras $A_{1 L}^{\nu \lambda}(\nu, \lambda= \pm 1)$ fit into a Hopf algebra extension

$$
1 \rightarrow\left(\mathbf{k} C_{2}\right)^{*} \rightarrow A_{1 L}^{\nu \lambda} \rightarrow \mathbf{k} D_{2 L} \rightarrow 1
$$

where $C_{2}$ is the cyclic group of order 2 and $D_{2 L}$ is the dihedral group of order $2 L$ (See $[4,7,8,11]$ for more precise information). So, the Hopf algebra $A_{1 L}^{\nu \lambda}$ can be regarded as a generalization of the 8-dimensional Hopf algebra introduced by Kac and Paljutkin [5]. (The Hopf algebra $A_{12}^{+-}$coincides with the 8-dimensional Kac-Paljutkin Hopf algebra.)

TheOrem 4 (S. Suzuki). (1) If $L \geq 3$, then the set of braidings of $A_{N L}^{\nu \lambda}$ is

$$
\left\{\sigma_{\alpha \beta} \mid \alpha, \beta \in \mathbf{k}^{\times},(\alpha \beta)^{N}=\nu,\left(\alpha \beta^{-1}\right)^{L}=\lambda\right\} .
$$

Here, $\sigma_{\alpha \beta}(x, y)$ is defined as in the following table.

\begin{tabular}{c|cccc}
$x \backslash y$ & $x_{11}$ & $x_{12}$ & $x_{21}$ & $x_{22}$ \\
\hline$x_{11}$ & 0 & 0 & 0 & 0 \\
$x_{12}$ & 0 & $\alpha$ & $\beta$ & 0 \\
$x_{21}$ & 0 & $\beta$ & $\alpha$ & 0 \\
$x_{22}$ & 0 & 0 & 0 & 0
\end{tabular}

(2) If $L=2$, then the set of braidings of $A_{N 2}^{\nu \lambda}$ is

$$
\left\{\sigma_{\alpha \beta} \mid \alpha, \beta \in \mathbf{k}^{\times},(\alpha \beta)^{N}=\nu,\left(\alpha \beta^{-1}\right)^{2}=\lambda\right\} \cup\left\{\tau_{\alpha \beta}^{\lambda} \mid \alpha, \beta \in \mathbf{k}^{\times}, \alpha^{2}=\beta^{2}, \alpha^{2 N}=1\right\} .
$$

Here, $\sigma_{\alpha \beta}(x, y)$ and $\tau_{\alpha \beta}^{\lambda}(x, y)$ are defined as in the following tables. (The left-hand side corresponds to $\sigma_{\alpha \beta}$ and the right-hand side corresponds to $\tau_{\alpha \beta}^{\lambda}$.)

\begin{tabular}{c|cccc}
$x \backslash y$ & $x_{11}$ & $x_{12}$ & $x_{21}$ & $x_{22}$ \\
\hline$x_{11}$ & 0 & 0 & 0 & 0 \\
$x_{12}$ & 0 & $\alpha$ & $\beta$ & 0 \\
$x_{21}$ & 0 & $\beta$ & $\alpha$ & 0 \\
$x_{22}$ & 0 & 0 & 0 & 0
\end{tabular}

\begin{tabular}{c|cccc}
$x \backslash y$ & $x_{11}$ & $x_{12}$ & $x_{21}$ & $x_{22}$ \\
\hline$x_{11}$ & $\alpha$ & 0 & 0 & $\beta$ \\
$x_{12}$ & 0 & 0 & 0 & 0 \\
$x_{21}$ & 0 & 0 & 0 & 0 \\
$x_{22}$ & $\lambda \beta$ & 0 & 0 & $\alpha$
\end{tabular}

REmark. Let $\nu, \lambda, N$ be as above. We consider a coideal $I^{\lambda}$ of $\mathcal{T}(C)$ defined by

$$
I^{\lambda}:=\mathbf{k}\left(X_{11} X_{22}-X_{22} X_{11}\right)+\mathbf{k}\left(X_{12} X_{21}-\lambda X_{21} X_{12}\right)+\sum_{i-j \neq l-m} \mathbf{k} X_{i j} X_{l m} .
$$

We set

$$
B^{\lambda}:=\mathcal{T}(C) /\left\langle I^{\lambda}\right\rangle
$$

Let $J_{N}^{\nu}$ be the coideal of $B^{\lambda}$ defined by

$$
J_{N}^{\nu}:=\mathbf{k}\left(x_{11}^{2}-x_{22}^{2}\right)+\mathbf{k}\left(x_{12}^{2}-x_{21}^{2}\right)+\mathbf{k}\left(x_{11}^{2 N}+\nu x_{12}^{2 N}-1\right) .
$$

Then the quotient bialgebra $B^{\lambda} / J_{N}^{\nu}$ is isomorphic to $A_{N 2}^{\nu \lambda}$. 
TheOREM 5. (1) The braided Hopf algebra $\left(A_{N L}^{\nu \lambda}, \sigma_{\alpha \beta}\right)$ has a coribbon element if and only if $N=1$.

Furthermore, the set of coribbon elements of $\left(A_{1 L}^{\nu \lambda}, \sigma_{\alpha \beta}\right)$ is $\left\{\theta_{\alpha}, \theta_{-\alpha}\right\}$, where $\theta_{\alpha}$ and $\theta_{-\alpha}$ are defined as in the following table.

\begin{tabular}{c|cccc}
$x$ & $x_{11}$ & $x_{12}$ & $x_{21}$ & $x_{22}$ \\
\hline$\theta_{\alpha}$ & $\alpha$ & 0 & 0 & $\alpha$ \\
$\theta_{-\alpha}$ & $-\alpha$ & 0 & 0 & $-\alpha$
\end{tabular}

(2) The set of coribbon elements of the braided Hopf algebra $\left(A_{N 2}^{\nu \lambda}, \tau_{\gamma \delta}^{\lambda}\right)$ is $\left\{\theta_{\omega} \mid \omega \in\right.$ $\mathbf{k}$ is a $2 N$-th root of unity\}, where $\theta_{\omega}$ is defined by

$$
\theta_{\omega}\left(x_{11}\right)=\theta_{\omega}\left(x_{22}\right)=\omega, \quad \theta_{\omega}\left(x_{12}\right)=\theta_{\omega}\left(x_{21}\right)=0 .
$$

We need some lemmas to prove Theorem 5 .

Lemma 6 (S. Suzuki). Let $\alpha, \beta$ be elements in $\mathbf{k}^{\times}$.

(1) Let $\sigma_{\alpha \beta}$ be the Yang-Baxter form on $C$ defined as in the following table.

\begin{tabular}{c|cccc}
$x \backslash y$ & $x_{11}$ & $x_{12}$ & $x_{21}$ & $x_{22}$ \\
\hline$x_{11}$ & 0 & 0 & 0 & 0 \\
$x_{12}$ & 0 & $\alpha$ & $\beta$ & 0 \\
$x_{21}$ & 0 & $\beta$ & $\alpha$ & 0 \\
$x_{22}$ & 0 & 0 & 0 & 0
\end{tabular}

Then $\sigma_{\alpha \beta}$ can be extended uniquely to a braiding on $B$. We denote the braiding on $B$ by $\sigma_{\alpha \beta}$ again.

(2) Let $\tau_{\alpha \beta}^{\lambda}$ be the Yang-Baxter form on $C$ defined as in the following table.

\begin{tabular}{c|cccc}
$x \backslash y$ & $x_{11}$ & $x_{12}$ & $x_{21}$ & $x_{22}$ \\
\hline$x_{11}$ & $\alpha$ & 0 & 0 & $\beta$ \\
$x_{12}$ & 0 & 0 & 0 & 0 \\
$x_{21}$ & 0 & 0 & 0 & 0 \\
$x_{22}$ & $\lambda \beta$ & 0 & 0 & $\alpha$
\end{tabular}

Then $\tau_{\alpha \beta}^{\lambda}$ can be extended uniquely to a braiding on $B^{\lambda}$. We denote the braiding on $B^{\lambda}$ by $\tau_{\alpha \beta}^{\lambda}$ again.

Lemma 7. Let $\omega$ be an element in $\mathbf{k}^{\times}$. We define a $\mathbf{k}$-linear form $\theta_{\omega}: C \rightarrow \mathbf{k}$ by

$$
\theta_{\omega}\left(x_{i j}\right)=\delta_{i j} \omega, \quad i, j=1,2 .
$$

(1) Let $\sigma_{\alpha \beta}$ be the braiding on $B$ given in Lemma 6 . Then $\theta_{\omega}$ can be extended uniquely to the coribbon element of the braided bialgebra $\left(B, \sigma_{\alpha \beta}\right)$. We denote the coribbon element on $B$ by $\theta_{\omega}$ again.

(2) Let $\tau_{\alpha \beta}^{\lambda}$ be the braiding on $B^{\lambda}$ given in Lemma 6 . Then $\theta_{\omega}$ can be extended uniquely to the coribbon element of the braided bialgebra $\left(B^{\lambda}, \tau_{\alpha \beta}^{\lambda}\right)$. We denote the coribbon element on $B^{\lambda}$ by $\theta_{\omega}$ again.

Proof. (1) Since $\theta_{\omega} * \theta_{\omega^{-1}}=\theta_{\omega^{-1}} * \theta_{\omega}=\varepsilon, \theta_{\omega}$ is invertible. Furthermore, $\theta_{\omega}$ satisfies the equation 


$$
\sum_{k=1}^{2} \theta_{\omega}\left(x_{i k}\right) x_{k j}=x_{i j}=\sum_{k=1}^{2} \theta_{\omega}\left(x_{k j}\right) x_{i k}
$$

Hence, by Theorem 2, $\theta_{\omega}$ can be extended to a coribbon element $\tilde{\theta}_{\omega}$ of the braided bialgebra $\left(M\left(C, \sigma_{\alpha \beta}\right), \tilde{\sigma}_{\alpha \beta}\right)$.

We consider the bialgebra homomorphism $\tilde{\iota}: M\left(C, \sigma_{\alpha \beta}\right) \rightarrow B$ induced from the inclusion $\iota: C \hookrightarrow B$. By Corollary 3, it is enough to show that

$$
\tilde{\theta}_{\omega}(\operatorname{Ker} \tilde{\iota})=0 .
$$

- If $\alpha^{2} \neq \beta^{2}$, then $\tilde{\iota}: M\left(C, \sigma_{\alpha \beta}\right) \rightarrow B$ is an isomorphism, so (1) is satisfied.

- If $\alpha^{2}=\beta^{2}$, then Ker $\tilde{\imath}$ is generated by $\mathcal{S}:=\left\{x_{i j} x_{l m} \mid i-j \not \equiv l-m(\bmod 2)\right\}$. The condition (1) is equivalent to

$$
\tilde{\theta}_{\omega}(S)=0
$$

By definition of $\sigma_{\alpha \beta}$ and $\theta_{\omega}$, we have

$$
\begin{aligned}
\tilde{\theta}_{\omega}\left(x_{i j} x_{l m}\right) & =\sum_{k, t, h, s=1}^{2} \tilde{\sigma}_{\alpha \beta}^{-1}\left(x_{i k}, x_{l t}\right) \tilde{\theta}_{\omega}\left(x_{k h}\right) \tilde{\theta}_{\omega}\left(x_{t s}\right) \tilde{\sigma}_{\alpha \beta}^{-1}\left(x_{s m}, x_{h j}\right) \\
& =\omega^{2} \tilde{\sigma}_{\alpha, \beta}^{-1}\left(x_{i, i+1}, x_{l, l+1}\right) \tilde{\sigma}_{\alpha \beta}^{-1}\left(x_{l+1, m}, x_{i+1, j}\right) \\
& =\omega^{2} \delta_{l m} \delta_{i j} \tilde{\sigma}_{\alpha \beta}^{-1}\left(x_{i, i+1}, x_{l, l+1}\right) \tilde{\sigma}_{\alpha \beta}^{-1}\left(x_{l+1, l}, x_{i+1, i}\right) .
\end{aligned}
$$

If $i-j \not \equiv l-m(\bmod 2)$, then $\delta_{l m} \delta_{i j}=0$. Therefore, we see that $\tilde{\theta}_{\omega}\left(x_{i j} x_{l m}\right)=0$, and whence $\tilde{\theta}_{\omega}(\mathcal{S})=0$.

(2) As in the proof of Part (1), we see that $\theta_{\omega}$ can be extended to a coribbon element $\tilde{\theta}_{\omega}$ of the braided bialgebra $\left(M\left(C, \tau_{\alpha \beta}^{\lambda}\right), \tilde{\tau}_{\alpha \beta}^{\lambda}\right)$.

Let $\tilde{\iota}: M\left(C, \tau_{\alpha \beta}^{\lambda}\right) \rightarrow B^{\lambda}$ be the bialgebra homomorphism induced from the inclusion $\iota: C \hookrightarrow B^{\lambda}$. Then, we have the following.

- If $\alpha^{2} \neq \lambda \beta^{2}$, then $\tilde{\iota}: M\left(C, \tau_{\alpha \beta}^{\lambda}\right) \rightarrow B^{\lambda}$ is an isomorphism.

- If $\alpha^{2}=\lambda \beta^{2}$, then Ker $\tilde{\iota}$ is generated by $\mathcal{S}:=\left\{x_{i j} x_{l m} \mid i-j \not \equiv l-m(\bmod 2)\right\}$.

- $\tilde{\theta}_{\omega}\left(x_{i j} x_{l m}\right)=\omega^{2} \delta_{l m} \delta_{i j}\left(\tilde{\tau}_{\alpha \beta}^{(\lambda)}\right)^{-1}\left(x_{i i}, x_{l l}\right)\left(\tilde{\tau}_{\alpha \beta}^{\lambda}\right)^{-1}\left(x_{l l}, x_{i i}\right)$.

By using these facts, by the same argument as in the proof of Part (1), we can prove Part (2). This completes the proof.

LEMMA 8. Let $\alpha, \beta, \omega$ be elements in $\mathbf{k}^{\times}$.

(1) Let $\theta_{\omega}$ be the coribbon element of $\left(B, \sigma_{\alpha \beta}\right)$ given in Lemma 7(1). We suppose that $(\alpha \beta)^{N}=\nu$ and $\left(\alpha \beta^{-1}\right)^{L}=\lambda$. Then $\theta_{\omega}$ induces a coribbon element of the bialgebra $\left(A_{N L}^{\nu \lambda}, \sigma_{\alpha \beta}\right)$ if and only if $N=1, \omega= \pm \alpha$.

(2) Let $\theta_{\omega}$ be the coribbon element of $\left(B^{\lambda}, \tau_{\alpha \beta}^{\lambda}\right)$ given in Lemma 7(2). We suppose that $\alpha^{2}=\beta^{2}$ and $\alpha^{2 N}=1$. Then $\theta_{\omega}$ induces a coribbon element of the bialgebra $\left(A_{N 2}^{\nu \lambda}, \tau_{\alpha \beta}^{\lambda}\right)$ if and only if $\omega^{2 N}=1$.

Proof. (1) We suppose that $(\alpha \beta)^{N}=\nu$ and $\left(\alpha \beta^{-1}\right)^{L}=\lambda$. Then we have:

$\theta_{\omega}$ induces a coribbon element of $\left(B /\left\langle J_{N L}^{\nu \lambda}\right\rangle, \sigma_{\alpha \beta}\right) \Leftrightarrow\left\{\begin{array}{l}(\mathrm{i}) \theta_{\omega}\left(x_{11}^{2 N}+\nu x_{12}^{2 N}-1\right)=0 \\ \left(\text { ii) } \theta_{\omega}\left(\chi_{1}^{L}-\chi_{2}^{L}\right)=0\right. \\ \text { (iii) } \theta_{\omega}\left(-\lambda \eta_{1}^{L}+\eta_{2}^{L}\right)=0 .\end{array}\right.$ 
Since

$$
\Delta\left(x_{i j}^{m}\right)=x_{i 1}^{m} \otimes x_{1 j}^{m}+x_{i 2}^{m} \otimes x_{2 j}^{m}
$$

for $m \geq 1$ and $i, j=1,2$, we have

$$
\begin{aligned}
& \theta_{\omega}\left(x_{11}^{m}\right)=\sigma_{\alpha \beta}^{-1}\left(x_{12}^{m-1}, x_{12}\right) \theta_{\omega}\left(x_{22}^{m-1}\right) \theta_{\omega}\left(x_{22}\right) \sigma_{\alpha \beta}^{-1}\left(x_{21}, x_{21}^{m-1}\right), \\
& \theta_{\omega}\left(x_{22}^{m}\right)=\sigma_{\alpha \beta}^{-1}\left(x_{21}^{m-1}, x_{21}\right) \theta_{\omega}\left(x_{11}^{m-1}\right) \theta_{\omega}\left(x_{11}\right) \sigma_{\alpha \beta}^{-1}\left(x_{12}, x_{12}^{m-1}\right), \\
& \theta_{\omega}\left(x_{12}^{m}\right)=\sigma_{\alpha \beta}^{-1}\left(x_{12}^{m-1}, x_{12}\right) \theta_{\omega}\left(x_{21}^{m-1}\right) \theta_{\omega}\left(x_{21}\right) \sigma_{\alpha, \beta}^{-1}\left(x_{12}, x_{12}^{m-1}\right)=0 .
\end{aligned}
$$

If $m$ is even, then

$$
\sigma_{\alpha \beta}^{-1}\left(x_{12}^{m-1}, x_{12}\right)=\sigma_{\alpha \beta}^{-1}\left(x_{21}, x_{21}^{m-1}\right)=\alpha^{-\frac{m}{2}} \beta^{-\left(\frac{m}{2}-1\right)} .
$$

If $m \geq 3$ is odd, then

$$
\sigma_{\alpha \beta}^{-1}\left(x_{21}^{m-1}, x_{21}\right)=\sigma_{\alpha \beta}^{-1}\left(x_{12}, x_{12}^{m-1}\right)=0 .
$$

Thus, we have

$$
\theta_{\omega}\left(x_{11}^{2 N}+\nu x_{12}^{2 N}\right)=\omega \alpha^{-2 N} \beta^{-(2 N-2)} \theta_{\omega}\left(x_{22}^{2 N-1}\right)= \begin{cases}0 & \text { if } N \geq 2 \\ \omega^{2} \alpha^{-2} & \text { if } N=1\end{cases}
$$

It follows that

$$
\text { (i) } \Leftrightarrow N=1 \text { and } \omega^{2}=\alpha^{2} \text {. }
$$

Now, we suppose $N=1$. For $m \geq 1$ we consider the following elements in $B$.

$$
\begin{aligned}
\chi_{11}^{m} & :=\underbrace{x_{11} x_{22} x_{11} \cdots \cdots}_{m}, \chi_{22}^{m}:=\underbrace{x_{22} x_{11} x_{22} \cdots \cdots}_{m}, \\
\chi_{12}^{m}: & =\underbrace{x_{12} x_{21} x_{12} \cdots \cdots}_{m}, \chi_{21}^{m}:=\underbrace{x_{21} x_{12} x_{21} \cdots \cdots}_{m} .
\end{aligned}
$$

Then the following formulae hold for $i, j=1,2$.

$$
\begin{gathered}
\Delta\left(\chi_{i j}^{m}\right)=\chi_{i 1}^{m} \otimes \chi_{1 j}^{m}+\chi_{i 2}^{m} \otimes \chi_{2 j}^{m}, \\
\sigma_{\alpha \beta}^{-1}\left(\chi_{11}^{m}, x_{i j}\right)=\sigma_{\alpha \beta}^{-1}\left(\chi_{22}^{m}, x_{i j}\right)=\sigma_{\alpha \beta}^{-1}\left(x_{i j}, \chi_{11}^{m}\right)=\sigma_{\alpha \beta}^{-1}\left(x_{i j}, \chi_{22}^{m}\right)=0, \\
\left\{\begin{array}{l}
\sigma_{\alpha \beta}^{-1}\left(\chi_{12}^{m}, x_{i j}\right)=\delta_{j, m+i} \alpha^{-m(j-1)} \beta^{-m(2-j)} \\
\sigma_{\alpha \beta}^{-1}\left(\chi_{21}^{m}, x_{i j}\right)=\delta_{j, m+i} \alpha^{-m(2-j)} \beta^{-m(j-1)}, \\
\sigma_{\alpha \beta}^{-1}\left(x_{i j}, \chi_{12}^{m}\right)=\delta_{i, m+j} \alpha^{-m(2-i)} \beta^{-m(i-1)}, \\
\sigma_{\alpha \beta}^{-1}\left(x_{i j}, \chi_{21}^{m}\right)=\delta_{i, m+j} \alpha^{-m(i-1)} \beta^{-m(2-i)} .
\end{array}\right.
\end{gathered}
$$

Here, we consider the indices of the Kronecker deltas modulo 2.

Using these equations, we have

$$
\begin{aligned}
\theta_{\omega}\left(\chi_{11}^{m}\right) & =\theta_{\omega}\left(x_{11} \chi_{22}^{m-1}\right) \\
& =\sum_{i, j=1,2} \sigma_{\alpha \beta}^{-1}\left(x_{1 i}, \chi_{21}^{m-1}\right) \theta_{\omega}\left(x_{i j}\right) \theta_{\omega}\left(\chi_{11}^{m-1}\right) \sigma_{\alpha \beta}^{-1}\left(\chi_{12}^{m-1}, x_{j 1}\right) \\
& =\omega \sum_{i=1,2} \theta_{\omega}\left(\chi_{11}^{m-1}\right) \delta_{1, i+m-1} \beta^{-(m-1)} \delta_{1, i+m-1} \beta^{-(m-1)} \\
& =\omega \theta_{\omega}\left(\chi_{11}^{m-1}\right) \beta^{-2(m-1)}
\end{aligned}
$$




$$
\begin{aligned}
\theta_{\omega}\left(\chi_{12}^{m}\right) & =\omega \sum_{i=1,2} \theta_{\omega}\left(\chi_{12}^{m-1}\right) \delta_{1, i+m-1} \beta^{-(m-1)} \delta_{2, i+m-1} \beta^{-(m-1)} \\
& =0 .
\end{aligned}
$$

By a similar calculation, we have

$$
\theta_{\omega}\left(\chi_{22}^{m}\right)=\omega \theta_{\omega}\left(\chi_{22}^{m-1}\right) \beta^{-2(m-1)}, \quad \theta_{\omega}\left(\chi_{21}^{m}\right)=0 .
$$

Thus, the condition (iii) is trivially satisfied. Since

$$
\begin{aligned}
\theta_{\omega}\left(\chi_{11}^{L}\right) & =\omega \beta^{-2(L-1)} \theta_{\omega}\left(\chi_{11}^{L-1}\right)=\omega^{2} \beta^{-2\{(L-1)+(L-2)\}} \theta_{\omega}\left(\chi_{11}^{L-2}\right)=\cdots \cdots \\
& =\omega^{L-1} \beta^{-2\{(L-1)+(L-2)+\cdots+1\}} \theta_{\omega}\left(\chi_{11}^{1}\right) \\
& =\omega^{L} \beta^{-L(L-1)}, \\
\theta_{\omega}\left(\chi_{22}^{L}\right) & =\omega^{L} \beta^{-L(L-1)},
\end{aligned}
$$

the condition (ii) is also trivially satisfied.

(2) We suppose that $\alpha^{2}=\beta^{2}$ and $\alpha^{2 N}=1$. Then we have:

$$
\theta_{\omega} \text { induces a coribbon element of }\left(B^{\lambda} /\left\langle J_{N}^{\nu}\right\rangle, \tau_{\alpha \beta}^{\lambda}\right) \Leftrightarrow\left\{\begin{array}{l}
(\mathrm{i}) \theta_{\omega}\left(x_{11}^{2 N}+\nu x_{12}^{2 N}-1\right)=0, \\
\text { (ii) } \theta_{\omega}\left(x_{11}^{2}-x_{22}^{2}\right)=0, \\
\text { (iii) } \theta_{\omega}\left(x_{12}^{2}-x_{21}^{2}\right)=0 .
\end{array}\right.
$$

Since

$$
\tilde{\theta}_{\omega}\left(x_{i j}^{2}\right)=\left(\tilde{\tau}_{\alpha \beta}^{\lambda}\right)^{-1}\left(x_{i i}, x_{i i}\right) \tilde{\theta}_{\omega}\left(x_{i j}\right) \tilde{\theta}_{\omega}\left(x_{i j}\right)\left(\tilde{\tau}_{\alpha \beta}^{\lambda}\right)^{-1}\left(x_{j j}, x_{j j}\right)=\tilde{\theta}_{\omega}\left(x_{i j}\right)^{2} \beta^{2},
$$

the conditions (ii) and (iii) are automatically satisfied. Since

$$
\left(\tau_{\alpha \beta}^{\lambda}\right)^{-1}\left(x_{j j}^{m-1}, x_{j j}\right)=\left(\tau_{\alpha \beta}^{\lambda}\right)^{-1}\left(x_{j j}, x_{j j}^{m-1}\right)=\alpha^{m-1}
$$

for $j=1,2$ and $m \geq 1$, it follows that

$$
\begin{aligned}
\theta_{\omega}\left(x_{11}^{m}\right) & =\left(\tau_{\alpha \beta}^{\lambda}\right)^{-1}\left(x_{11}^{m-1}, x_{11}\right) \theta_{\omega}\left(x_{11}^{m-1}\right) \theta_{\omega}\left(x_{11}\right)\left(\tau_{\alpha \beta}^{\lambda}\right)^{-1}\left(x_{11}, x_{11}^{m-1}\right) \\
& =\omega \gamma^{2(m-1)} \theta_{\omega}\left(x_{11}^{m-1}\right) \theta_{\omega}\left(x_{22}^{m}\right), \\
\theta_{\omega}\left(x_{22}^{m}\right) & =\left(\tau_{\alpha \beta}^{\lambda}\right)^{-1}\left(x_{22}^{m-1}, x_{22}\right) \theta_{\omega}\left(x_{22}^{m-1}\right) \theta_{\omega}\left(x_{22}\right)\left(\tau_{\alpha \beta}^{\lambda}\right)^{-1}\left(x_{22}, x_{22}^{m-1}\right) \\
& =\omega \alpha^{2(m-1)} \theta_{\omega}\left(x_{22}^{m-1}\right), \\
\theta_{\omega}\left(x_{12}^{m}\right) & =\left(\tau_{\alpha \beta}^{\lambda}\right)^{-1}\left(x_{11}^{m-1}, x_{11}\right) \theta_{\omega}\left(x_{12}^{m-1}\right) \theta_{\omega}\left(x_{12}\right)\left(\tau_{\alpha \beta}^{\lambda}\right)^{-1}\left(x_{22}, x_{22}^{m-1}\right) \\
& =0 .
\end{aligned}
$$

By induction on $m$, we have

$$
\theta_{\omega}\left(x_{11}^{m}\right)=\theta_{\omega}\left(x_{22}^{m}\right)=\omega^{m} \alpha^{2((m-1)+(m-2)+\cdots+1)}=\omega^{m} \alpha^{m(m-1)} .
$$

Therefore, we have

$$
\text { (i) } \Leftrightarrow \omega^{2 N} \alpha^{2 N(2 N-1)}=1 \quad \Leftrightarrow \quad \omega^{2 N}=1 .
$$

This completes the proof.

Proof of Theorem 5. Let $\theta \in\left(A_{N L}^{\nu \lambda}\right)^{*}$ be a coribbon element of $\left.\left(A_{N L}^{\nu \lambda}\right\rangle, \sigma_{\alpha \beta}\right)$, where $\alpha, \beta \in \mathbf{k}^{\times}$satisfy $(\alpha \beta)^{N}=\nu,\left(\alpha \beta^{-1}\right)^{L}=\lambda$, and $\sigma_{\alpha \beta}$ is the braiding given in Theorem 4 . Since $L \geq 2$,

$$
\left\{x_{11}, x_{12}, x_{21}=\nu x_{12}^{2 N} x_{21}, x_{22}=x_{11}^{2 N} x_{22}\right\}
$$


is a system of linearly independent vectors in $A_{N L}^{\nu \lambda}$. Hence, comparing the equations

$$
\theta\left(x_{11}\right) x_{11}+\theta\left(x_{12}\right) x_{21}=\theta\left(x_{11}\right) x_{11}+\theta\left(x_{21}\right) x_{12}
$$

and

$$
\theta\left(x_{11}\right) x_{12}+\theta\left(x_{12}\right) x_{22}=\theta\left(x_{12}\right) x_{11}+\theta\left(x_{22}\right) x_{12},
$$

we see that

$$
\theta\left(x_{12}\right)=\theta\left(x_{21}\right)=0, \quad \theta\left(x_{11}\right)=\theta\left(x_{22}\right) .
$$

We put $\omega:=\theta\left(x_{11}\right)=\theta\left(x_{22}\right)$. Then $\omega \neq 0$, since $\theta$ is invertible. Moreover, $\theta=\theta_{\omega} \circ \pi$, where $\theta_{\omega}$ is the coribbon element of $\left(B, \sigma_{\alpha \beta}\right)$, and $\pi: B \rightarrow B /\left\langle J_{N L}^{\nu \lambda}\right\rangle$ is the natural projection. By Lemma 8, it follows that $N=1$ and $\omega= \pm \alpha$.

Next, let $\theta \in\left(A_{N L}^{\nu \lambda}\right)^{*}$ be a coribbon element of $\left(A_{N L}^{\nu \lambda}, \tau_{\alpha \beta}^{\lambda}\right)$, where $\alpha, \beta \in \mathbf{k}^{\times}$satisfy $\alpha^{2}=\beta^{2}, \alpha^{2 N}=1$, and $\tau_{\alpha \beta}^{\lambda}$ is the braiding given in Theorem 4 . With the same argument as in the proof of the first part, we see that

$$
\theta\left(x_{12}\right)=\theta\left(x_{21}\right)=0, \quad \theta\left(x_{11}\right)=\theta\left(x_{22}\right) .
$$

We put $\omega:=\theta\left(x_{11}\right)=\theta\left(x_{22}\right)$. Then $\omega \neq 0$, since $\theta$ is invertible. Moreover, $\theta=\theta_{\omega} \circ \pi^{\prime}$, where $\theta_{\omega}$ is the coribbon element of $\left(B^{\lambda}, \tau_{\alpha \beta}^{\lambda}\right)$, and $\pi^{\prime}: B^{\lambda} \rightarrow B^{\lambda} /\left\langle J_{N}^{\nu}\right\rangle \cong A_{N 2}^{\nu \lambda}$ is the natural projection. Therefore, by Lemma 8 , it follows that $\omega^{2 N}=1$.

Since any coribbon element $\theta=\theta_{\omega}$ satisfies $\theta \circ S=\theta$, it is a coribbon element for a braided Hopf algebra. This completes the proof.

Acknowledgments. The author is grateful to Professor A. Masuoka for having suggested the problems in this article and for explaining Suzuki's results. He would also like to thank Professor Y. Doi and Professor M. Takeuchi for encouragement, and Professors P. Hajac, W. Pusz and S. L. Woronowicz for hospitality at the Banach Center.

\section{References}

[1] Y. Doi, Braided bialgebras and quadratic bialgebras, Comm. Algebra 21 (1993), 1731-1749.

[2] V. G. Drinfel'd, Quantum groups, in: Proceedings of the International Congress of Mathematics, Berkeley, CA, 1987, 798-820.

[3] T. Hayashi, Coribbon Hopf (face) algebras generated by lattice models, J. Algebra 233 (2000), 614-641.

[4] M. Izumi and H. Kosaki, Kac algebras arising from composition of subfactors: General theory and classification, preprint.

[5] G. I. Kac and V. G. Paljutkin, Finite ring groups, Trans. Moscow Math. Soc. 1966, AMS, 1967, 251-294 (original Russian paper: Trudy Moskov. Mat. Obshch. 15 (1966), 224-261).

[6] L. H. Kauffman, Knots and physics, World Scientific, 1991.

[7] A. Masuoka, Cocycle deformations and Galois objects for some cosemisimple Hopf algebras of finite dimension, in: Contemp. Math. 267, AMS, 2000, 195-214.

[8] A. Masuoka, Hopf algebra extensions and cohomology, preprint to appear in: New Directions in Hopf Algebras, S. Montgomery and H.-J. Schneider (eds.), MSRI Publ. 43, Cambridge Univ. Press.

[9] D. E. Radford, Solutions to the quantum Yang-Baxter equation arising from pointed bialgebras, Trans. Amer. Math. Soc. 343 (1994), 455-477. 
[10] N. Yu. Reshetikhin and V. G. Turaev, Ribbon graphs and their invariants derived from quantum groups, Commun. Math. Phys. 127 (1990), 1-26.

[11] S. Suzuki, A family of braided cosemisimple Hopf algebras of finite dimension, Tsukuba J. Math. 22 (1998), 1-29.

[12] M. E. Sweedler, Hopf Algebras, Benjamin, New York, 1969.

[13] M. Takeuchi, A short course on quantum matrices, preprint, Mathematisches Institut, Ludwig-Maximilians-Universität, 1998.

[14] M. Takeuchi and D. Tambara, A new one-parameter family of $2 \times 2$ matrix bialgebras, Hokkaido Math. J. 21 (1992), 405-419. 